\title{
Angiotomografía-3D y la microcirugía de los aneurismas: Avance preliminar
}

\author{
JULIO RAMÍREZ \\ Servicio de Neurocirugía, Hospital Edgardo Rebagliati - Essalud. Facultad de Medicina, UNMSM.
}

\begin{abstract}
RESUMEN
OBJETIVO: Determinar si la pan-angiografía cerebral con sustracción digital convencional y la angiotomografía tridimensional (AngioTac-3D) permiten visualizar los aneurismas tridimensionalmente en pacientes con hemorragia subaracnoidea (HSA) por ruptura de aneurisma. MATERIAL Y MÉTODOS: Se presenta los primeros 10 casos de pacientes varones o mujeres que han ingresado con HSA aguda o "tardía" en quienes se ha realizado pan-angiografía cerebral con sustracción digital convencional y la AngioTac-3D y fueron objeto de la microcirugía correspondiente. RESULTADOS: El uso de la angiotomografia tridimensional con reconstrucción de superficie nos permite visualizar las estructuras vasculares, venosas y óseas, girando las mismas en 360 grados; por ello es necesario la presencia del neurocirujano durante el examen, para contar con imágenes que permitan un criterio quirúrgico. CONCLUSIÓN: El examen no invasivo angiotomografía tridimensional permite diagnosticar la hemorragia subaracnoidea, como se comprueba en estos primeros casos.
\end{abstract}

Palabras clave: Aneurisma; angiografía de substracción digital; microcirugía.

\begin{abstract}
ANGIOTOMOGRAPHY - 3D AND ANEURISMS MICROSURGERY: PRELIMINARY REPORT

SUMMARY

OBJECTIVE: To determine if cerebral pan-angiography with conventional digital substraction and AngioTac-3D allow tridimensional visualization of aneurisms in patients with acute subarachnoid hemorrhage (ASH) due to rupture of an aneurism. MATERIAL AND METHODS: We present the first 10 cases of men and women who were admitted with either acute or "late" ASH to whom cerebral pan-angiography with conventional digital substraction and 3D-AngioTac were performed followed by microsurgery. RESULTS: Use of tridimensional angiotomography with surface reconstruction allows us to see vascular, venous and bony structures, rotating them in 360 degrees. It follows the need that the neurosurgeon be present during the procedure in order that images lead the surgical criterium. CONCLUSION: The non-invasive tridimensional angiotomography allows diagnosis of subarachnoid hemorrhage, as seen in these preliminary report.
\end{abstract}

Key words: Aneurism; angiography, digital subtraction; microsurgery.

\section{INTRODUCCIÓN}

En recientes estudios realizados para evaluar pacientes con patología vascular, la importancia de la aplicación de la angiotomografía tridimensional (AngioTac-3D) es reportada sucesivamente por varios investigadores, como

\footnotetext{
Correspondencia:

Dr. Julio Ramírez Villavicencio

Av. Euterpe 153

Lima 3, Perú

E-mail:jrmercupe@yahoo.com
}

Harbaugh, en 1995 ( $^{1}$ ) y luego Zouaoui, en 1997 $\left.{ }^{2}\right)$. Al mismo tiempo, la utilidad de la tomografía axial computarizada y la angiografía cerebral en los casos de ruptura de aneurismas han sido reconocidas por Nakajima y col, en $1998\left({ }^{3}\right)$, y por Anderson y col, en $1999\left({ }^{4}\right)$.

En razón de éstas y otras publicaciones, últimamente venimos aplicando esta metodología en nuestros pacientes portadores de aneurismas congénitos. Así, cuando se produce la ruptura aneurismática y se presenta el cuadro de hemo- 
rragia subaracnoidea (HSA), el examen inicial es la tomografía axial computarizada (TAC) cerebral sin contraste $\left({ }^{5}\right)$. En forma inmediata o posterior al episodio, según sea el caso, la panangiografía cerebral convencional con sustracción digital nos permitirá el diagnóstico topográfico biplanar del aneurisma.

El avance vertiginoso de la tecnología en neu-roimágenes, entre ellas, la angiotomografía tridimensional (AngioTac-3D), nos permite estudiar la arquitectura vascular cerebral y sus relaciones con las estructuras venosas y óseas, dándonos mayor conocimiento tridimensional de las mismas. La aplicación de estos exámenes y su seguimiento, ha motivado nuestra inquietud, por lo que realizamos el presente trabajo preliminar.

\section{MATERIAL Y MÉTODOS}

En nuestro hospital contamos con un tomógrafo helicoidal Somaton Plus 4, en el que se realiza la angiotomografía tridimensional a los pacientes con HSA; en la Unidad Radiológica Vascular e Intervencionista (URVI) se realiza la pan-angiografía cerebral convencional con sustracción digital.

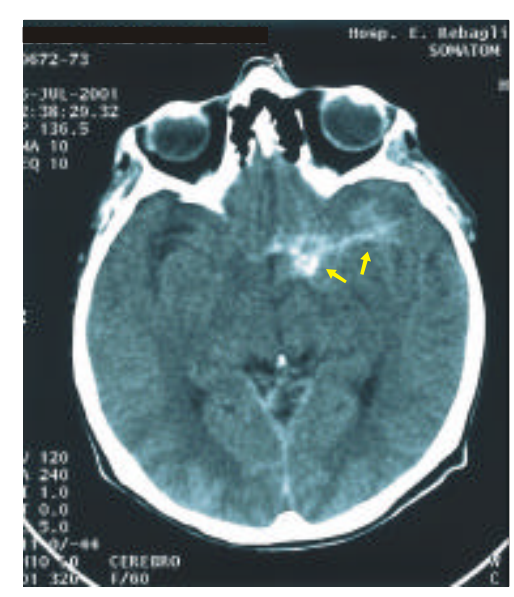

Figura 1.- LAC. Tomografía axial computarizada sin contraste: Hemorragia subaracnoidea en cisternas basales (flechas).
De un total de 10 pacientes con HSA (Figura 1), en los últimos dos meses se realizó los estudios de angiografía cerebral con sustracción digital convencional y la AngioTac-3D; se exceptuó un caso, que inició con cuadro de compresión del III N. Presentamos los casos demostrativos, que llegaron por emergencia o transferidos de otros hospitales con HSA "espontánea" reciente o "tardía”.

En los 10 primeros pacientes adultos, de sexos y edades diferentes, admitidos en nuestro hospital, su condición clínica fue determinada con la escala de Hunt y Hess (HH) y la TAC cerebral con la escala de Fisher (Tabla 1).

La localización de los aneurismas fue realizada por el mismo observador y confirmada posteriormente en el acto operatorio. No se midió el tamaño de los aneurismas.

\section{RESULTADOS}

Los pacientes con HSA, a los cuales se ha realizado la panangiografía cerebral convencional mostrando uno o más aneurismas, también han sido observados con la AngioTac-3D, con reconstrucción de superficie en todos los casos, y fueron confirmados en el acto operatorio (Figuras 2-5).

Tabla 1.- Distribución de pacientes con aneurisma cerebral.

\begin{tabular}{lcllll}
\hline Nombre & Edad & Sexo & HH & Fischer & \multicolumn{1}{c}{ Distribución } \\
\hline MCP & 59 & F & II & 2 & ACoA \\
JHN & 65 & M & II & 2 & ACM derecha \\
ARG & 69 & M & III & 4 & ACI-ACM izquierda \\
LAC & 60 & F & II & 2 & ACI izquierda \\
& & & & & (2 aneurismas) \\
AJC & 53 & M & II & 2 & ACoP izquirda \\
ACS & 45 & M & I & 2 & ACI-ACM derecha \\
ERN & 44 & F & I & 1 & ACoP derecha \\
MFR & 70 & F & II & 2 & ACM derecha \\
JRB & 43 & M & IV & 4 & ACM izquierda \\
WMB & 37 & M & I & 1 & ACI derecha - \\
& & & & & ACM izquierda \\
\hline
\end{tabular}

ACoA = Arteria comunicante anterior

$A C M=$ Arteria cerebral media

$A C I=$ Arteria carótida interna

ACoP $=$ Arteria comunicante posterior 

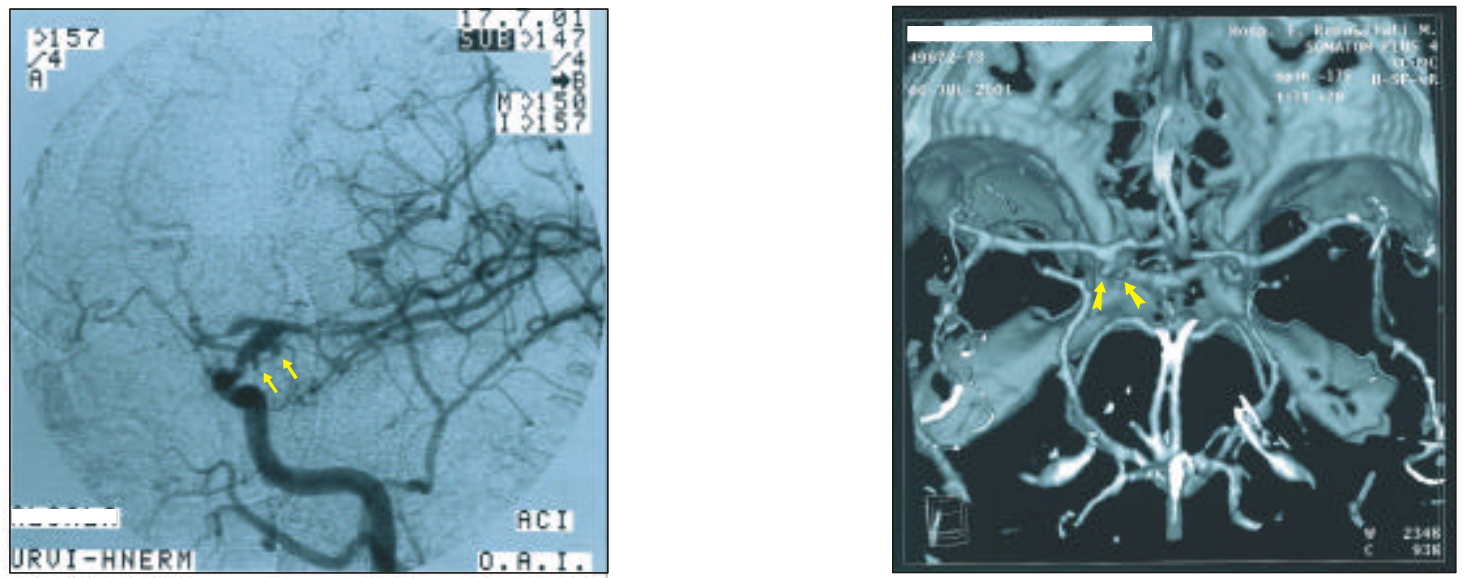

Figura 2.- LAC. A la derecha: Angiotac-3D aneurismas en arteria carótida interna izquierda (flechas). A la izquierda: Angiografía convencional (flechas).
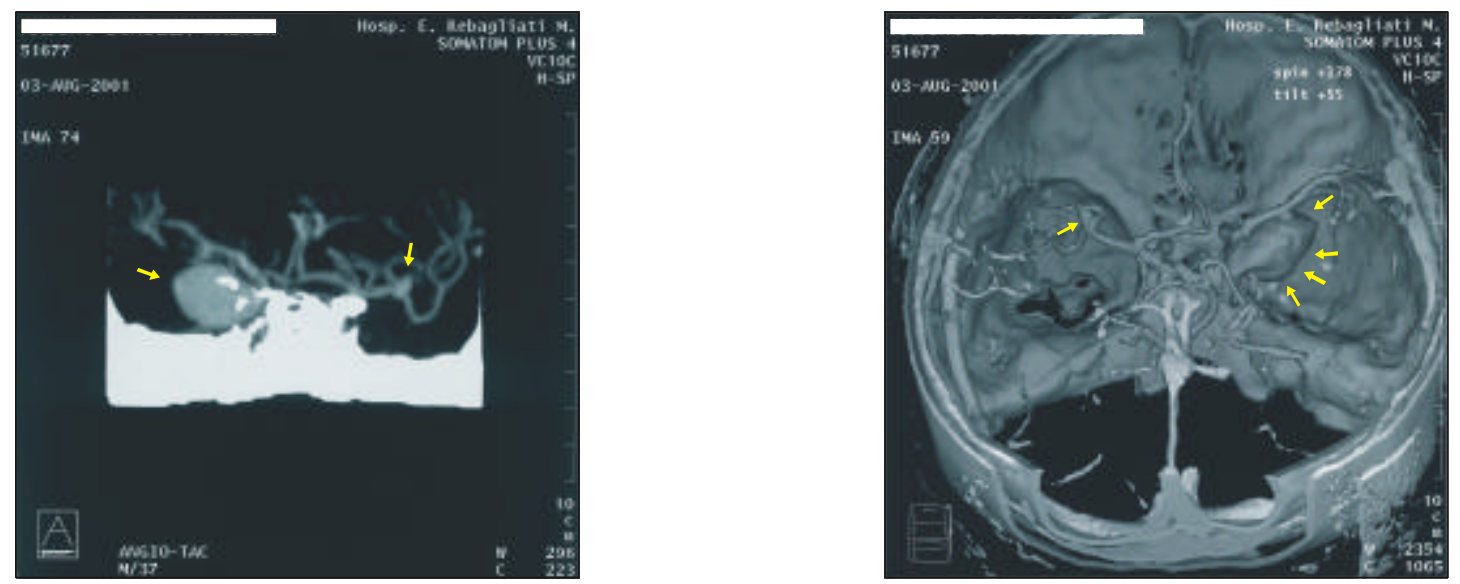

Figura 3.- WMB. Angiotomografía-3D. A la derecha: Aneurisma gigante de arteria carótida interna (flechas). A la izquierda: Aneurisma de bifurcación de arteria cerebral media (flechas).
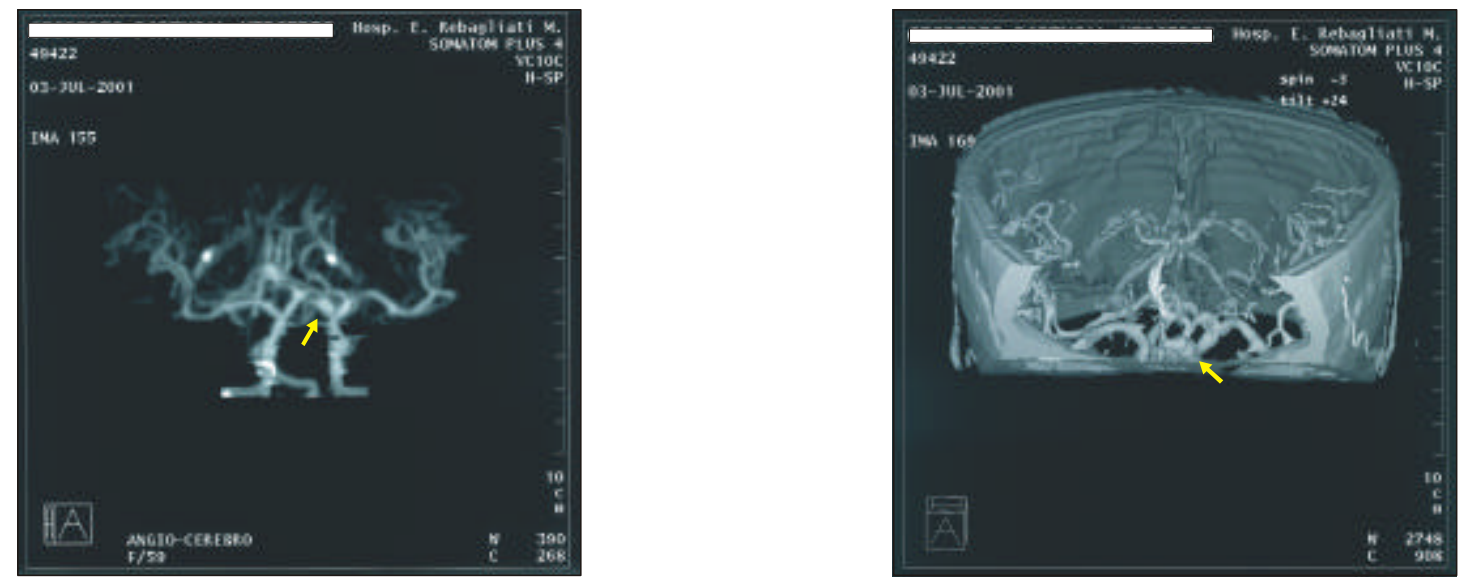

Figura 4.- MCP. Angiotac-3D. Ambos: Aneurisma de arteria comunicante anterior. (flechas). 

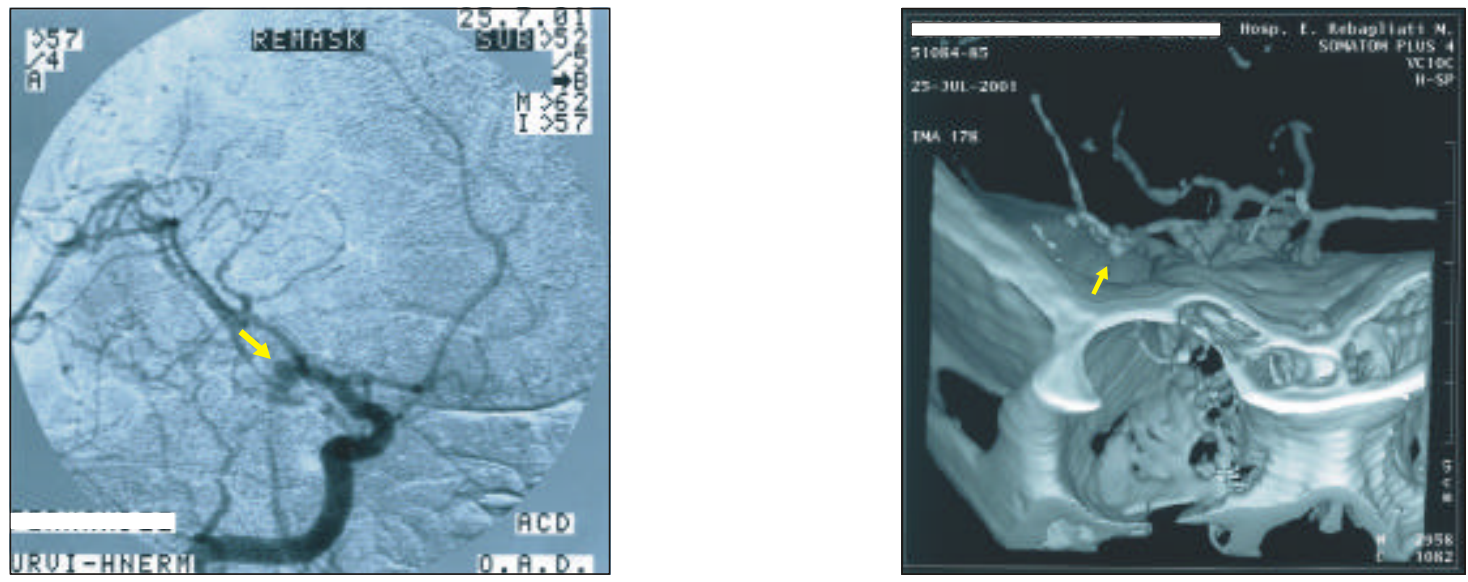

Figura 5.- MFR. A la derecha: Angiografía convencional muestra aneurisma de arteria cerebral media (flecha). A la izquierda: Angiotac-3D idem (flecha).

Se requiere de neurocirujanos especializados en las patologías vasculares y un equipo y personal dedicado a los exámenes de la AngioTac3D para una adecuada intrepretación y planificación estratégica del acto operatorio.

\section{DISCUSIÓN}

Se ha publicado en los últimos años trabajos relacionados con AngioTac-3D, en tomógrafos de mayor resolución, con personal especialmente dedicado a la patología vascular cerebral. Los resultados son inobjetables, hasta se plantea la posibilidad de realizar directamente la microcirugía de aneurismas, sin recurrir al examen invasivo consistente en la pan-angiografía cerebral convencional.

La microcirugía de aneurismas luego de las AngioTac-3D, sin la necesidad de realizar las angiografías convencionales, es una posibilidad actual. Algunos servicios ya la están realizando. Estamos cerca de ellos.

El presente reporte preliminar de los primeros diez casos, nos permite concluir que, en los pacientes con HSA, valorados con la escala de Hunt y Hess y Fisher, a quienes se realizó la pan-angiografía cerebral con sustracción digital convencional y la AngioTac-3D, estos procedi- mientos permiten visualizar los aneurismas tridimensionalmente, en todos los casos; mejor aún en las reconstrucciones de superficie, correlacionándose con las estructuras óseas y dando al neurocirujano una mayor y mejor visión para la microcirugía de aneurismas. Esta posibilidad tecnológica nos presenta un futuro prometedor, que evitará recurrir al uso de exámenes invasivos.

\section{BIBLIOGRAFÍA}

1. Anderson GB, Steinke DE, Petruk KC, Ashforth R, Findlay JM. Computed tomographic angiography versus substraction angiography for the diagnosis and early treatment of ruptured intracraneal aneurysms. Neurosurgery 1999; 45:1315-22.

2. Drake CG. Report of the World Federation of Neurological Surgeons Committee on a Universal Subarachnoid Hemorrhage Grading Scale. J Neurosurg 1988; 68: 985-6.

3. Harbaugh RE, Schlusselberg DS, Jeffery R, Hayden S, Cromwell LD, Pluta D, et al. Three-dimensional computed tomographic angiography in the preoperative evaluation of cerebrovascular lesions. Neurosurgery 1995; 36: 320-7.

4. Nakajima Y, Yoshimine T, Yoshida H, Sakashita K, Okamoto $M$, Kishikawa $M$, et al. Computerized tomography angiography of ruptured cerebral aneurysms: factors affecting time to maximum contrast concentration. J Neurosurg 1998; 88: 663-9.

5. Zouaoui A, Sahel M, Marro B, Clemenceau S, Dargent $\mathbf{N}$, Bitar A, et al. Three-dimensional computed tomographic angiography in detection of cerebral aneurysms in acute subarachnoid hemorrhage. Neurosurgery 1997; 41: 125-30. 